\title{
The influence of Saffman lift force on nanoparticle concentration distribution near a wall
}

\author{
Xu Zheng and Zhanhua Silber-Li $i^{\text {a) }}$ \\ State Key Laboratory of Nonlinear Mechanics, Institute of Mechanics, CAS, Beijing 100190, \\ People's Republic of China
}

(Received 4 May 2009; accepted 2 August 2009; published online 25 September 2009)

\begin{abstract}
The lift force on a spherical nanoparticle near a wall in micro/nanofluidics has not received sufficient attention so far. In this letter the concentration of $\phi 200 \mathrm{~nm}$ particles is measured at $0.25-2.0 \mu \mathrm{m}$ to a wall in a microchannel with pressure-driven de-ionized water flow (pressure gradient $0-2000 \mathrm{kPa} / \mathrm{m}$ ). The measured data show the influence of the lift force on the nanoparticle concentration distribution. By introducing the Saffman lift force into the Nernst-Planck equation near a wall, we find that the lift force is dominant at the range of $2<z^{+}<6\left(z^{+}=z / 2 r, r\right.$ is the particle radius, $z$ is the distance from the wall). (C) 2009 American Institute of Physics.

[doi:10.1063/1.3237159]
\end{abstract}

With the development of near-field detection technique, the nonuniform concentration distribution of nanoparticles very near a wall in micro/nanofluidics attracts attention. Hartman Kok et al. ${ }^{1}$ used attenuated total reflection-infrared spectroscopy to measure the concentration distribution of particles $(\sim \phi 100 \mathrm{~nm})$ at a distance of around $1 \mu \mathrm{m}$ from the wall and proposed an exponential equation to describe the distribution. Bouzigues et al. ${ }^{2}$ used total internal reflection fluorescence microscopy to measure the concentration distribution of $\phi 20 \mathrm{~nm}$ nanoparticles close to the wall in thermal equilibrium and found that the electrostatic force is dominant within $200 \mathrm{~nm}$ of the wall. Zheng and Silber- $\mathrm{Li}^{3}$ observed that the concentration distributions of $\phi 50$ and $\phi 200 \mathrm{~nm}$ nanoparticle tracers were not uniform within $1 \mu \mathrm{m}$ to wall, and it would lead to a deviation of the measured velocity by microparticle imaging velocimetry/particle tracking velocimetry.

When particles flow through a channel, their concentration distribution will become biased close to wall due to lateral migration. ${ }^{4}$ In a macroscale flow $[\operatorname{Re} \sim O(10-1000)]$, a moving particle near a wall is affected by the lift forces including the Magnus force due to particle rotation and the Saffman force due to near wall shear. ${ }^{5}$ The Saffman force can be expressed as ${ }^{6}$

$$
F_{L}=K \mu V r^{2}(\dot{\gamma} / \nu)^{1 / 2},
$$

where constant $K=81.2, r$ is the particle radius, $V=u_{f}-u_{p}$ is the relative velocity, $u_{p}$ is the velocity of the particle and $u_{f}$ is that of the fluid in the streamline through the particle center, $\dot{\gamma}$ is the shear rate, $\mu$ is the dynamic viscosity, and $\nu$ is the kinetic viscosity. In micro/nanofluidics, with much diminished typical flow dimensions, the shear effect could be very strong near a wall. However, the shear influence on the concentration distribution of nanoparticles is still not well studied. Furthermore, in the region within $1 \mu \mathrm{m}$ of a wall, the electrostatic effect should be also considered.

Therefore, we measure the concentration distribution of $\phi 200 \mathrm{~nm}$ Polystyrene fluorescent particles at $250 \mathrm{~nm}-2 \mu \mathrm{m}$ of a wall in a microchannel, under different driven pressures $(\Delta p=0-20 \mathrm{kPa})$, to investigate the influence of the lift force due to shear near a wall. The experimental results are also analyzed based with the NernstPlanck equation where the Saffman lift force is introduced.

The measurements were performed on a fluorescent inverted microscope (Olympus IX71), equipped with a 100 $\times / \mathrm{NA}=1.35$ objective (Fig. 1). A piezotransducer (Physik Instrument LVPZT E665) was mounted under the objective to control the position of the focus plane with a precision of $10 \mathrm{~nm}$. An electron-multiplying charge-coupled device (CCD) (EMCCD, Andor DV885) was used to record images with an $80 \times 80 \mu \mathrm{m}$ view field. The $\phi 200 \mathrm{~nm}$ fluorescent polystyrene particles (Duke Scientific Corporation, density $1.05 \mathrm{~g} / \mathrm{cm}^{3}$ ) were excited by $532 \mathrm{~nm}$ green light to emit red light at $610 \mathrm{~nm}$. These particles were diluted into de-ionized (DI) water (Millipore@) at a volume concentration of 5 $\times 10^{-5}$.

The microchannel was made of poly-dimethylsiloxane bonded by a coverglass $\left(160 \mu \mathrm{m}\right.$ thick, contact angle $\left.25^{\circ}\right)$. The DI water with nanoparticles flowed through a microchannel $(55 \times 20 \mu \mathrm{m}, 1 \mathrm{~cm}$ in length $)$ driven by nitrogen gas, under five pressures $\Delta p=0,2,5,10$, and $20 \mathrm{kPa}$ (pressure gradient $0-2000 \mathrm{kPa} / \mathrm{m}$ ). The corresponding wall shear rate $\dot{\gamma}$ ranged approximately from 0 to $1.9 \times 10^{4} \mathrm{~s}^{-1}$. The vertical measurement positions were $z=0.25,0.5,0.75,1.0$, 1.5 , and $2.0 \mu \mathrm{m}$, controlled by the piezotransducer. A thresh-

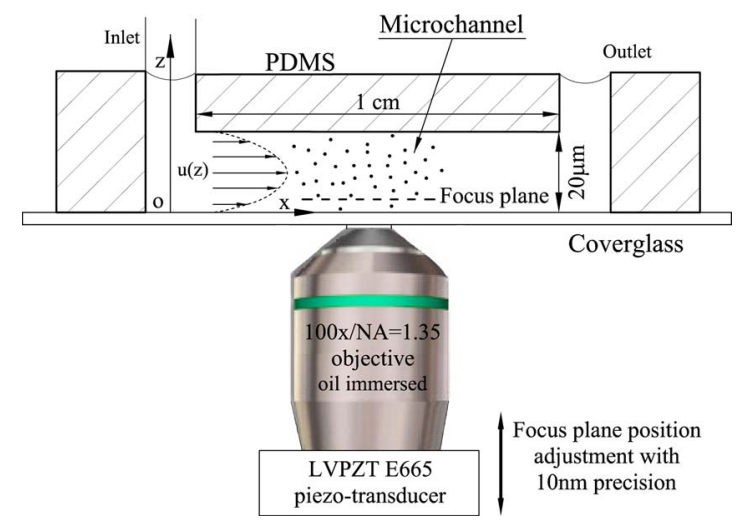

FIG. 1. (Color online) Schematic diagram of the experimental setup.

${ }^{a)}$ Electronic mail: lili@imech.ac.cn. 


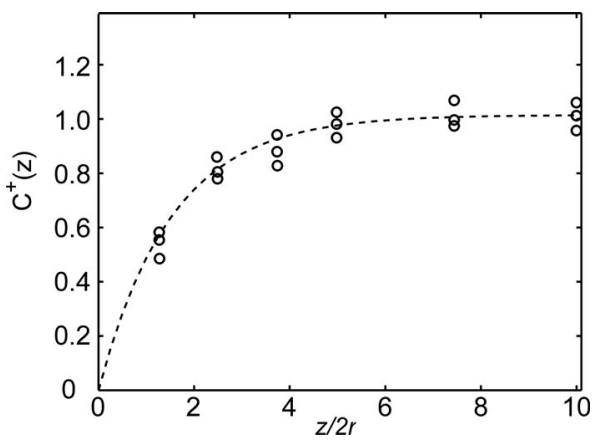

FIG. 2. The $200 \mathrm{~nm}$ nanoparticle concentration distribution close to the wall in DI water when $\Delta p=0$. The circles are the experimental data, and the dashed line is the exponentially fitted curve.

old of the grayscale value, $80 \%$ of the maximum grayscale value in the images, was chosen to filter out the out-of-plane particles $^{7}$ and achieve an approximately $0.5 \mu \mathrm{m}$ effective focus plane thickness. According to a method proposed by Joseph and Tabeling, ${ }^{8}$ the wall position $(z=0)$ is determined by the particles adsorbed to the wall, and the $z$ position is adjusted by the piezoelectric transducer. The uncertainty is estimated to be $25-40 \mathrm{~nm}$. Also the measurements were carried out at three horizontal positions along a channel. The temperature was $23-25{ }^{\circ} \mathrm{C}$.

By counting the number of particles in images recorded at different $z$ locations, the concentration distribution near the wall can be obtained. For each $z$ location the measured concentration out of more than 20000 nanoparticles was counted. The nanoparticle concentration distribution in DI water close to the wall was first measured in a steady state $(\Delta p=0)$. The following dimensionless variables are adopted: $z^{+}=z / 2 r, C^{+}\left(z^{+}\right)=C\left(z^{+}\right) / C_{0}$, where $C_{0}$ is the concentration far from the wall. Figure 2 shows that the measured data are obviously nonuniform below $z^{+}=6$. Following Hartman Kok's equation, ${ }^{1}$ an exponential function is used to fit the experimental data,

$$
C^{+}\left(z^{+}\right)=-A\left(e^{-B z^{+}}-1\right),
$$

where $A$ and $B$ are two constants obtained from data fitting. When $\Delta p=0, A=1.015$. $C^{+}$will approach unity far from the wall, so $A \approx 1$ is reasonable. $B=0.653$ represents how biased the concentration distribution is.

The measurements were made in a similar manner for $\Delta p=2,5,10$, and $20 \mathrm{kPa}(\operatorname{Re}=0.25-2.5)$ using the same channel. The experiments were repeated three times using different channels. The measured data and the fitted curves are shown in Fig. 3, and the constants $A$ and $B$ are given in
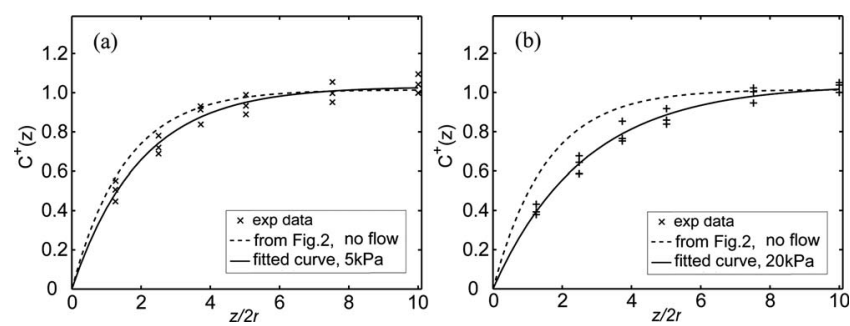

FIG. 3. The $200 \mathrm{~nm}$ nanoparticle concentration distribution close to the wall in DI water under different driven pressures [(a) $5 \mathrm{kPa}$; (b) $20 \mathrm{kPa}$. The corresponding wall shear rates $\dot{\gamma}$ are approximately in the range of 1900-19000 s ${ }^{-1}$.
TABLE I. The fitted coefficients A and B.

\begin{tabular}{llcccc}
\hline \hline$\Delta p(\mathrm{kPa})$ & 0 & 2 & 5 & 10 & 20 \\
$\dot{\gamma}\left(\mathrm{s}^{-1}\right)$ & 0 & 1900 & 4750 & 9500 & 19000 \\
$\mathrm{~A}$ & 1.015 & 0.998 & 1.031 & 1.023 & 1.040 \\
$\mathrm{~B}$ & 0.653 & 0.603 & 0.508 & 0.432 & 0.381 \\
\hline \hline
\end{tabular}

Table I. In the experiments, $B$ can reach 0.381 at $\Delta p$ $=20 \mathrm{kPa}$, which is significantly smaller than its value of 0.653 when $\Delta p=0$. The measurement shows that (a) as $\Delta p$ increases, $C^{+}$decreases when $z^{+}<7.5$, but it keeps constant when $z^{+}>7.5$. (b) The influence of shear on the concentration distribution is much more obvious at $2<z^{+}<5$ (Fig. 3).

To investigate the concentration distribution, an approach following Nernst-Planck equation is proposed. Considering the lift force, the electrostatic force, and the diffusion effect, the equation of the equilibrium state of a spherical particle normal to the wall is

$$
D \cdot \nabla C+C \mu_{e}\left(\nabla \phi-F_{L}\right)=0 .
$$

The term $D \cdot \nabla C$ is related to the particle diffusion, with $D$ being the diffusion coefficient of the particle. The second term is related to the electrostatic force $F_{E}=\nabla \phi$ and the lift force $F_{L}$, with $\mu_{e}=D / k_{B} T$ being the particle mobility. Electrostatic force $F_{E}$ is ${ }^{9}$

$$
F_{E}(z)=-16 \kappa \varepsilon r\left(\frac{k_{B} T}{e}\right)^{2} \operatorname{th}\left(\frac{e \psi_{w}}{4 k_{B} T}\right) \operatorname{th}\left(\frac{e \psi_{s}}{4 k_{B} T}\right) e^{-\kappa z},
$$

where $\varepsilon$ is the dielectric permittivity of water, $\kappa^{-1}$ is the Debye length, and $\psi_{w}$ and $\psi_{s}$ are the Stern potentials of wall and particle, respectively. Theoretically, the Debye length in DI water $(18.2 \mathrm{M} \Omega \cdot \mathrm{cm})$ is approximately $300 \mathrm{~nm}$. However in the actual case, it is just a few $100 \mathrm{~nm}$ due to a $p \mathrm{H}$ value deviated from $7 .^{10}$ Thus we may assume that $\kappa^{-1} \approx 150 \mathrm{~nm}$, $\psi_{w} \approx-50 \mathrm{mV}$, and $\psi_{s} \approx-20 \mathrm{mV}$, according to the literature. ${ }^{2}$ For the lift force, as an approximation, the Saffman force [Eq. (1)] may take its place. The relative velocity $V=u_{f}-u_{p}$ in Eq. (1) is calculated following Goldman's result, ${ }^{11^{p}}$

$$
u_{p} / u_{f}=1-\frac{5}{16}\left(\frac{r}{z}\right)^{3}
$$

where the Poiseuille velocity is used as $u_{f}$. Equation gives $u_{p} / u_{f}=0.995-0.9997$ at $z^{+}=2.5-5$.

With Eqs. (1), (4), and (5), Eq. (3) can be solved by integration with the boundary condition that $C(z)$ goes to $C_{0}$ at $z=h$. The solution is

$$
\begin{aligned}
C^{+}(z)= & \frac{C(z)}{C_{0}}=\exp \left\{-\frac{\phi}{k T}-\frac{2 A_{1}}{k T}\left[\sqrt{h-z}+\frac{h \sqrt{h-z}}{z}\right.\right. \\
& \left.\left.-\sqrt{h} \arctan h\left(\frac{\sqrt{h-z}}{\sqrt{h}}\right)\right]\right\},
\end{aligned}
$$

where $h$ is the half height of the channel, $A_{1}=12.7 \rho^{0.5} r^{5} /$ $\mu(-d p / d x)^{1.5}$. The results from Eq. (6) under $\Delta p=5$ and 20 $\mathrm{kPa}$ are shown in Fig. 4. Compared with the experimental data, the curves of Eq. (6) represent well the trend of concentration distribution at $z^{+}>2$ : (a) $C^{+}$decreases as $\dot{\gamma}$ increases. (b) The influence of shear is obvious at $2<z^{+}<6$. Beyond $z^{+}=6$, the near wall effects can be omitted. However at $z^{+}<2$, the experimental data should be retested by other 

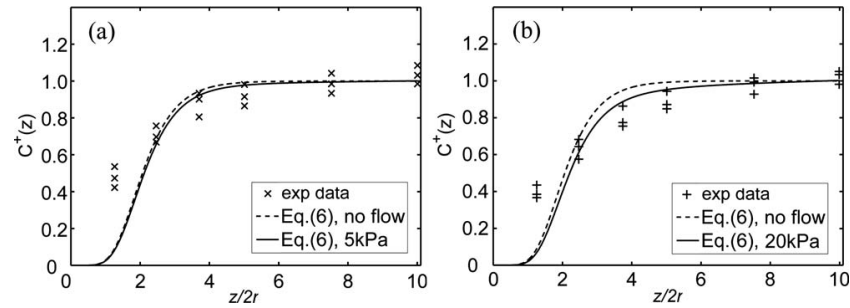

FIG. 4. A comparison between the solution of Eq. (6) (solid lines) and the experimental data (markers) under (a) $\Delta p=5 \mathrm{kPa}$ and (b) $\Delta p=20 \mathrm{kPa}$. The dash lines are the results from Eq. (6) when $\Delta p=0$.

near field techniques. A comparison between the Saffman force $F_{L}$ and the electrostatic force $F_{E}$ at $z^{+}=1-10$ is shown in Fig. 5. The electrostatic force should be dominant when $z^{+}<2$. When $z^{+}>3$, the influence of the lift force becomes significant. With the increase in $\dot{\gamma}$, the influence range of $F_{L}$ extends closer to the wall, as shown from the experiments.

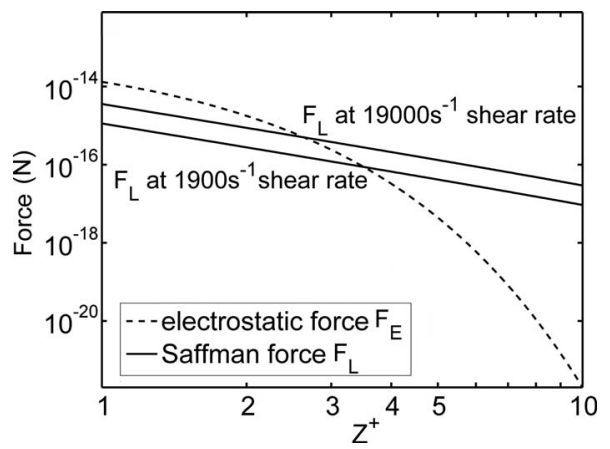

FIG. 5. A comparison between the electrostatic force (dashed line) and the Saffman lift force (solid lines) in the range of $z^{+}=1-10$. The wall shear rates $\dot{\gamma}$ are $1900-19000 \mathrm{~s}^{-1}$.
In summary, the concentration distributions of $\phi 200 \mathrm{~nm}$ nanoparticles close to a wall under different driven pressure gradients were measured. The experimental results show that the influence of lift force due to shear on the concentration distribution is much more significant at $2<z^{+}<5$. A theoretical analysis introducing the Saffman lift force into the Nernst-Planck equation near a wall is proposed and shows that the lift force is dominant at approximately $2<z^{+}<6$. Thus we can conclude that the Saffman force is a dominant factor for the nanoparticle concentration distribution in this range.

The authors gratefully thank the support of this work by the National Natural Science Foundation of China (Grant Nos. 10672172 and 10872203), the National Basic Research Program (Grant No. 2007CB714501) and the Hi-Tech Research and Development Program of China (Grant No. 2007AA04Z302).

${ }^{1}$ P. J. A. Hartman Kok, S. G. Kazarian, B. J. Briscoe, and C. J. Lawrence, J. Colloid Interface Sci. 280, 511 (2004).

${ }^{2}$ C. I. Bouzigues, P. Tabeling, and L. Bocquet, Phys. Rev. Lett. 101, 114503 (2008).

${ }^{3}$ X. Zheng and Z. H. Silber-Li, PIV09, Melbourne, Australia, 25-28 August (2009).

${ }^{4}$ L. G. Leal, Annu. Rev. Fluid Mech. 12, 435 (1980).

${ }^{5}$ J. P. Matas, J. F. Morris, and E. Guazzelli, Oil \& Gas Sci. \& Tech. - Revue de l'IFP 59, 59 (2004).

${ }^{6}$ P. G. Saffman, J. Fluid Mech. 22, 385 (1965).

${ }^{7}$ X. Zheng and Z. H. Silber-Li, Exp. Fluids 44, 951 (2008).

${ }^{8}$ P. Joseph and P. Tabeling, Phys. Rev. E 71, 035303 (2005).

${ }^{9}$ A. Banerjee and K. D. Kihm, Phys. Rev. E 72, 042101 (2005).

${ }^{10}$ H.-J. Butt, K. Graf, and M. Kappl, Physics and Chemistry of Interfaces (Wiley, Weinheim, 2003).

${ }^{11}$ A. J. Goldman, R. G. Cox, and H. Brenner, Chem. Eng. Sci. 22, 653 (1967). 